study telomere length was linked to the $\mathrm{X}$ chromosome ${ }^{9}$. The investigators found a correlation in telomere lengths of fathers and daughters and of mothers and daughters or sons but not of fathers and sons. Perhaps polymorphisms in the gene associated with dyskeratosis congenita on the $\mathrm{X}$ chromosome influence telomere maintenance. Thus, the $\mathrm{X}$ chromosome would influence telomere length heritability in a manner analogous to the anticipation phenomenon described by Vulliamy et al. ${ }^{3}$.

Several correlative studies support the idea that individuals with telomeres shorter than those of age-matched controls are prone to disease. For example, individuals with atherosclerotic heart disease have significantly shorter telomeres than healthy aged-matched controls ${ }^{10}$. Independent of age and mean arterial pressure, arterial stiffness and pulse pressure inversely correlate with TRF length in men ${ }^{11}$. In a retrospective study of archival cryopreserved white blood cells, telomere length was a primary independent predictor of overall mortality. In this study, mortality due to infectious disease was eight times greater in individuals (over the age of $60 \mathrm{y}$ ) whose blood-cell telomere length was in the lowest quartile (shortest telomeres) than in individuals whose telomeres were in the other quartiles ${ }^{12}$. Vulliamy et al. ${ }^{3}$ show that genetically inherited short telomeres in dyskeratosis congenita cause anticipation of disease. This supports the hypothesis that short telomeres due to a variety of causes (increased in cell turnover associated with chronic age-associated diseases, inflammatory processes, oxidative damage, genetic alterations in telomerase components) correlate with disease. Showing cause and effect will require showing that slowing down the rate of telomere loss or resetting the telomere clock reverses or delays the onset of disease ${ }^{13}$.

Because telomerase RNA is limiting in vivo in dyskeratosis congenita and telomeres are not maintained to age-appropriate lengths, is dyskeratosis congenita the perfect disease to test the therapeutic value of telomere rejuvenation?

\section{Treatment options}

One approach to test if telomere rejuvenation could affect the progression of disease in dyskeratosis congenita would be to isolate hematopoietic stem cells $\left(\mathrm{CD} 34^{+}\right)$from individuals with dyskeratosis congenita, expand them in the laboratory while transiently overexpressing the catalytic protein (TERT) component of telomerase (perhaps using adenoviral TERT that does not integrate into nuclear DNA) until telomeres become sufficiently elongated, and then return the rejuvenated stem-like cells to the affected individual. The obvious advantage of this approach is that these are the person's own cells, which would avoid problems of rejection. In addition, this could be done without ablating the person's own bone marrow cells (skewing of X-chromosome inactivation in women carrying the X-linked form of dyskeratosis congenita shows that progressive overgrowth of cells with longer telomeres is likely to occur). The ectopic expression of TERT may be sufficient to maintain or elongate telomeres in a wide variety of cells ${ }^{14,15}$. Safety and efficacy standards, as well as quality and control assurances, will need to be carefully considered before initiating such studies. But if this strategy for engineering telomeres in cells improves the health and longevity of individuals with dyskeratosis congenita, then it could be considered for treatment of other telomere-based proliferative deficiencies produced by disease or aging.

1. Mason, P.J. Bioessays 25, 126-133 (2003).

2. Shay, J.W. \& Wright, W.E. Science 286, 2284-2285 (1999)

3. Vulliamy, T. et al. Nat. Genet. 36, 447-449 (2004).

4. Collins, K. \& Mitchell, J.R. Oncogene 21, 564-579 (2002).

5. Fu, D. \& Collins, K. Mol. Cell 11, 1361-1372 (2003).

6. Hathcock, K.S. et al. Proc. Natl. Acad. Sci. USA 99, 3591-3596 (2002).

7. Slagboom, P.E., Droog, S. \& Boomsmas, D.I. Am J. Hum. Genet. 55, 876-882 (1994).

8. Wu, X. et al. J. Natl. Cancer Inst. 95, 1211-1218 (2003)

9. Nawrot, T.S., Staessen, J.A., Gardner, J.P. \& Aviv, A. Lancet 363, 507-510 (2004).

10. Samani, N.J., Boultby, R., Butler, R. Thompson, J.R. \& Goodall, A.H. Lancet 358, 472-473 (2001).

11. Benetos, A. et al. Hypertension 37, 381-385 (2000).

12. Cawthon, R.M., Smith, K.R., O'Brien, E. Sivatchenko, A. \& Kerber, R.A. Lancet 361, 393-395 (2003)

13. Shay, J.W. \& Wright, W.E. Nat. Biotechnol. 18, 22-23 (2000).

14. Bodnar, A.G. et al. Science 279, 349-352 (1998).

15. Morales, C.P. et al. Nat. Genet. 21, 115-118 (1999).

\title{
The spreading influence of chromatin modification
}

\author{
Mark Schlissel
}

\section{Specific post-translational histone modifications correlate with distinct patterns of gene activity. A new study of the mouse Dntt promoter shows that changes in gene activity are associated with local changes in histone modification that then spread outward in both directions, locking in the altered pattern of transcription.}

The mouse genome consists of about 3 billion bp of DNA divided into 20 chromosomes. Stretched out, this DNA would measure nearly a meter in length, but it all fits into a spherical nucleus only several microns in diameter. It is a challenge to understand how the genome can be wrapped so tightly yet remain accessible for precisely regulated gene

Mark Schlissel is in the Department of Molecular and Cell Biology at University of California, Berkeley, 439 LSA, Berkeley, California, USA.

e-mail:mss@uclink4.berkeley.edu expression. Meeting this challenge will require a detailed appreciation of the dynamics of chromatin structure. On page 502 of this issue, a new study by Ruey-Chyi Su and colleagues ${ }^{1}$ illustrates how localized changes in chromatin structure influence the regulated expression of a specific gene during mouse lymphocyte development.

\section{Chromatin represses transcription}

The most basic element of chromatin structure, the nucleosome, consists of an octamer of histones forming a central core and $146 \mathrm{bp}$ of DNA wrapped nearly twice around $\mathrm{it}^{2}$.
Successive nucleosomes are separated by 20-60 bp of DNA, often associated with a linker histone called H1. This 11-nM nucleosome fiber is then wrapped into a $30-\mathrm{nM}$ solenoidal structure. Our understanding of this, and subsequent higher orders of chromatin structure, is rudimentary.

Chromatin structure is a substantial barrier to the transcription machinery. Basal transcription factors, including the essential TFIID complex and RNA polymerase II, cannot recognize promoter sequences packaged in a nucleosome array ${ }^{3}$. Two processes cooperate to clear the way for gene activation ${ }^{4}$. The 
first, known as chromatin remodeling, slides nucleosomes out of the way to expose promoter elements. The second consists of the covalent modification of histones through the placement or removal of acetyl, methyl, ADPribosyl or phosphate groups on or from specific amino acids. Some of these histone modifications correlate with regulated gene activation or constitutive inactivity (the 'histone code') $)^{5}$. The study by Su et al. ${ }^{1}$ characterizes the changes in histone modifications that occur when an active gene undergoes developmentally regulated inactivation.

Dntt encodes an enzyme involved in the diversification of antigen receptor genes in developing lymphocytes ${ }^{6}$. It is active early in B- and T-cell development but is inactivated as lymphocytes mature. Previous work identified the Dntt promoter and defined an array of transcription factors regulating its activity. $\mathrm{Su}$ et al. compared two model systems in which an artificial stimulus promotes the differentiation of immature $\mathrm{T}$ cells in vitro, resulting in inactivation of Dntt expression.

They found that nucleosomes containing histone $\mathrm{H} 3$ modified by acetylation of lysine at position 9 (acetylated H3-Lys9) were enriched throughout a domain extending at least $10 \mathrm{~kb} 5^{\prime}$ and $3^{\prime}$ of the promoter in cultured immature thymocytes and in a thymic lymphoma cell line, VL3-3M2. After inducing differentiation, they observed the highly localized loss of this modification over the promoter region itself, associated temporally with inactivation of Dntt transcription. In primary thymocytes, this was followed over the ensuing $18 \mathrm{~h}$ by the gradual spread of this region of deacetylated chromatin in either direction away from the promoter. During this interval, they detected loss of another activation-associated modification, acetylation of $\mathrm{H} 3$ at Lys4, followed by the acquisition of methylation at H3-Lys9, again spreading from the promoter outwards. After $6-8 \mathrm{~h}$ of induction, the inactivation of transcription became irreversible. Notably, VL3-3M2 cells showed the same focal loss of acetylated H3Lys 9 at the promoter, but this modification did not spread outward and transcriptional inactivation remained reversible. Thus, Su et al. ${ }^{1}$ found that localized changes in histone modifications are associated with transient changes in gene activity, whereas permanent changes are associated with regional spread of a domain of chromatin modification.

\section{Who's calling the shots?}

Some transcription factors have, or recruit proteins that have, histone modification and remodeling activities (Fig. 1). Presumably, gene activation requires at least one such factor that can bind its recognition sequence within 'inactive' chromatin and recruit other factors that collaborate in altering local chromatin structure. These altered regions of chromatin would then expose binding sites for other factors, including the basal transcription machinery $^{3}$. Histone modifications may also be necessary to allow RNA polymerase to transit across nucleosomal DNA sequences ${ }^{7}$. When cells progress through development, the levels of various transcription factors might change and key target sites might be vacated. Alternatively, as might be the case with Dntt, negative transcription factors might compete with and displace activating factors, altering associated chromatin remodeling and modifying activities $^{8}$ (Fig. 1). The tendency of such alterations to occur locally and then spread globally is poorly understood. Perhaps local histone modification itself recruits additional modifying enzymes, leading to spread along the DNA. This idea, which gains support from studies in yeast and fruit flies ${ }^{9,10}$, implies that boundary elements limit the spread of such modified chromatin domains ${ }^{2}$.

The study by Su et al. ${ }^{1}$ also addresses the difference between transformed and primary immature T cells. Dntt inactivation was fully reversible in VL3-3M2 but not primary thymocytes, a difference that correlated with a failure of spreading in VL3-3M2 cells. Primary thymocytes did not undergo cell division in this culture system whereas transformed cells did. Perhaps cell cycle regulation or chromatin reassembly after replication is involved in the spread and reversibility of chromatin modification patterns. Alternatively, transformed cells might have different levels of the relevant modifying enzymes, perhaps as a consequence of the transformed phenotype. Also, whereas

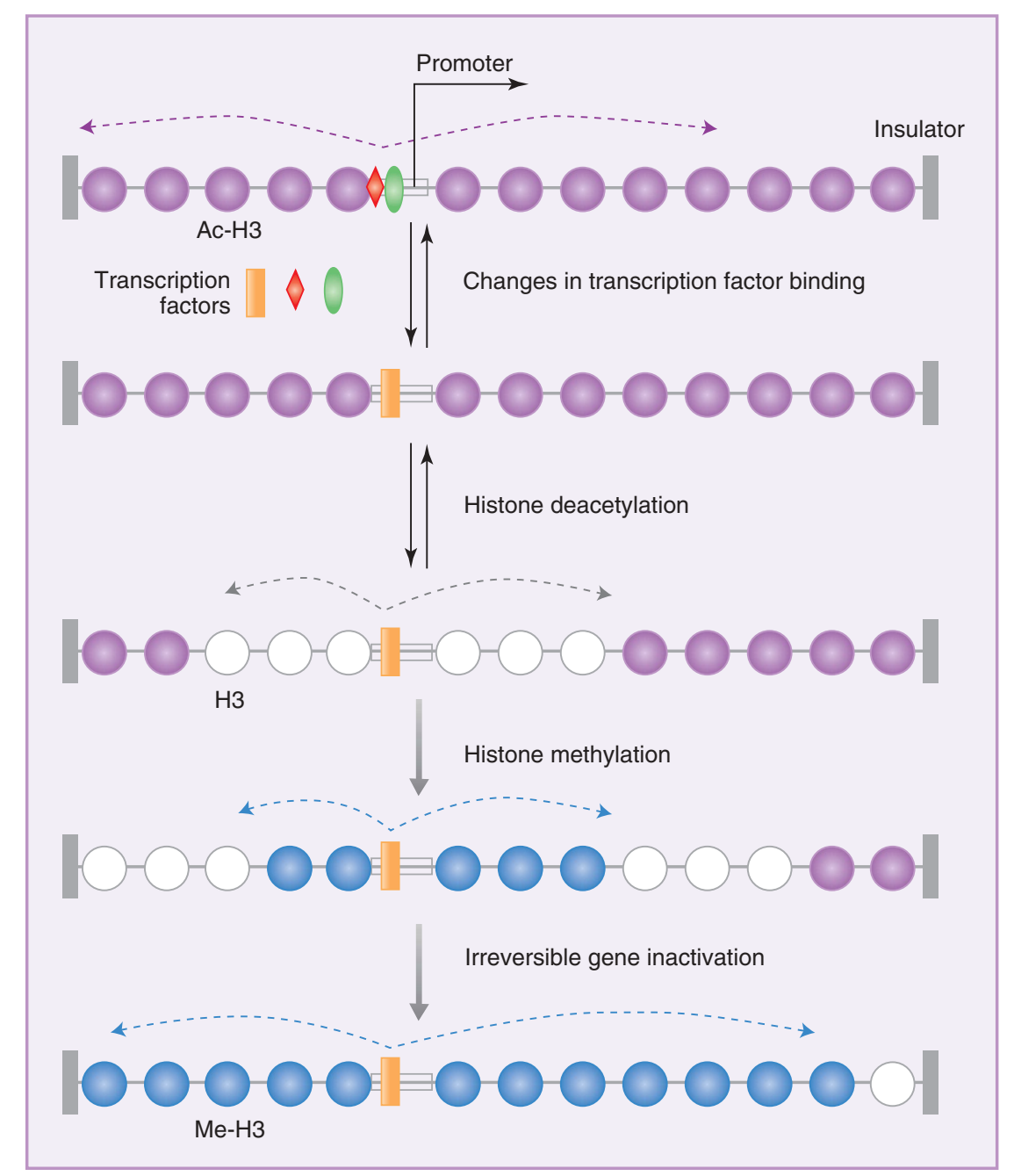

Figure 1 Transcription factors recruit chromatin modification enzymes, which in turn regulate chromatin structure in the vicinity of the promoter. Dashed lines indicate spread of chromatin changes outward from the promoter region. In this model, changes are reversible until nucleosomes are modified by histone methylation. Ac, acetylated; Me, methylated. 
acetylation can be reversed by various histone deacetylases, there are no known histone demethylases. Therefore, once a genomic region is methylated, modified nucleosomes must be replaced rather than altered to remove this epigenetic mark. Further work will be required to understand the mechanisms responsible for the spread of local histone modifications and the impact of these modifications on chromatin structure and transcriptional regulation.

1. Su, R.-C. et al. Nat. Genet. 36, 502-506 (2004).

2. Felsenfeld, G. \& Groudine, M. Nature 421, 448-453 (2003).

3. Struhl, K. Cel/ 98, 1-4 (1999).

4. Narlikar, G.J., Fan, H.Y. \& Kingston, R.E. Cell 108 475-487 (2002).
5. Jenuwein, T. \& Allis, C.D. Science 293, 1074-1080 (2001).

6. Benedict, C.L., Gilfillan, S., Thai, T.H. \& Kearney, J.F. Immunol. Rev. 175, 150-157 (2000).

7. Wittschieben, B.O. et al. Mol. Cell 4, 123-128 (1999).

8. Trinh, L.A. et al. Genes Dev. 15, 1817-1832 (2001).

9. Rusche, L.N., Kirchmaier, A.L. \& Rine, J. Annu. Rev. Biochem. 72, 481-516 (2003).

10. Ahmad, K. \& Henikoff, S. Cell 104, 839-847 (2001).

\title{
Src kinases in $\mathrm{Ph}^{+}$lymphoblastic leukemia
}

\author{
Michael Deininger
}

\author{
Philadelphia chromosome-positive leukemia may present as chronic myeloid leukemia or B-cell acute lymphoblastic \\ leukemia. A new study shows that certain Src kinases are necessary for the induction of B-cell acute lymphoblastic \\ leukemia but not chronic myeloid leukemia.
}

What is the basis of the tissue specificity of certain oncogenes? Are there differences in the frequency of certain mutations, perhaps related to differences in DNA repair, or is tissue specificity a question of the target cell having the right signaling network in place to allow for transformation by a specific oncogene? In the latter case, are the essential components of this network identical, regardless of the cell type? In Philadelphia chromosome-positive $\left(\mathrm{Ph}^{+}\right)$leukemia, caused by the $B C R-A B L 1$ translocation, this has been a longstanding question, as $\mathrm{Ph}^{+}$disease may present as one of two distinct entities, chronic myeloid leukemia (CML) or B-cell acute lymphoblastic leukemia (B-ALL). Clinical observations, mutational studies of $B C R-A B L 1$ and mouse leukemia models present a complex picture, and it has been difficult to identify differences in the use of downstream signaling pathways by Bcr-Abl. On page 453 of this issue, Yiguo $\mathrm{Hu}$ and colleagues ${ }^{1}$ show that certain Src kinases are essential to the ability of Bcr-Abl to induce B-ALL but not CML. Their results shed new light on an old discussion and provide a rationale for the use of Src kinase inhibitors to treat $\mathrm{Ph}^{+} \mathrm{B}-\mathrm{ALL}$.

\section{CML and $\mathrm{Ph}^{+} \mathbf{B}-\mathrm{ALL}$}

The Philadelphia chromosome was initially thought to be specific for $\mathrm{CML}^{2}$, a disease characterized by excessive proliferation of myeloid cells with mostly normal differentiation. The picture blurred when individuals

Michael Deininger is in the Center for Hematological Malignancies, Oregon Health \& Science University, Portland, Oregon, USA.

e-mail:deininge@ohsu.edu

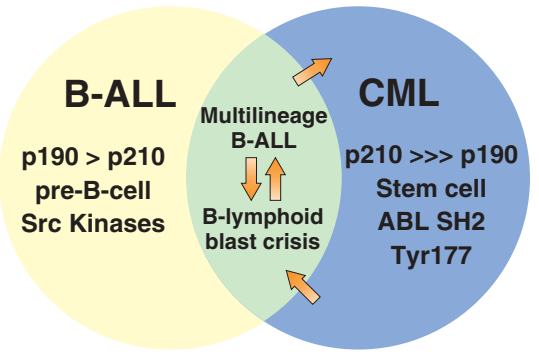

Figure 1 Induction of B-ALL versus CML by BcrAbl. p190, the shorter form, is practically restricted to B-ALL, whereas p210 is found in both conditions. As shown by Hu et al. ${ }^{1}$, certain Src kinases are required for induction of B-ALL, whereas several structural motifs in p210 BCR$A B L 1$ are required for or favor the induction of CML. It is not clear if multilineage B-ALL and lymphoid blast crisis are different presentations of the same entity or different diseases.

were reported with $\mathrm{Ph}^{+}$myeloproliferative disorders other than CML or acute leukemias, including ALL, a rapidly fatal disease of undifferentiated lymphoid cells ${ }^{3}$. Then it was determined that the blastic phase of CML could include lymphoid differentiation. Conversely, CML was diagnosed in some individuals after successful treatment of $\mathrm{Ph}^{+}$ ALL. This suggested the existence of 'genuine' $\mathrm{Ph}^{+} \mathrm{B}$-ALL as opposed to multilineage disease, perhaps representing CML diagnosed in lymphoid blast crisis ${ }^{4}$ (Fig. 1).

Is the phenotype of $\mathrm{Ph}^{+}$leukemia determined by features of Bcr-Abl, by the cell type that acquires the oncogene or by both? Clinically, the two common Bcr-Abl variants differ greatly in their capacity to induce CML but not B-ALL. The larger p210 Bcr-Abl variant is found in almost all individuals with CML and also in one-third of those with $\mathrm{Ph}^{+}$ B-ALL. In contrast, the p190 variant of Bcr$\mathrm{Abl}$ is present in two-thirds of those with $\mathrm{Ph}^{+}$ B-ALL and only rarely in $\mathrm{CML}^{5}$. Explanations include the weaker tyrosine kinase activity of p210 Bcr-Abl, which may consistently induce CML but only occasionally induce B-ALL. Alternatively, the GTP exchange and Rac GTPase functions present in p210 Bcr-Abl but not in p190 Bcr-Abl may be crucial for induction of CML but not B-ALL. In mice transplanted with bone marrow infected with $B C R-A B L 1$ retroviruses, motifs in the first 210 amino acids of $\mathrm{Bcr}$ (specifically, the coiledcoil domain and Tyr177) promote CML but not B-ALL 6 . Despite these hints, no signaling pathways specific for either type of disease had been definitively identified.

\section{A role for Src kinases}

$\mathrm{Hu}$ et al. ${ }^{1}$ show that the Src family kinases Lyn, Hck and Fgr are activated by Bcr-Abl in primary mouse B-lymphoblasts. They show that disease latency and survival were greatly extended in wild-type mice transplanted with $B C R-A B L 1$-infected marrow obtained from mice with homozygous deletions of at least two of these three Src kinases. In contrast, the induction of CML was not affected. CGP76030, a Src kinase inhibitor, significantly prolonged the survival of mice with BALL but not CML. Taken together, this is strong evidence that Lyn, Hck and Fgr are crucial to induction of B-ALL by Bcr-Abl but dispensable for induction of CML. Lack of one kinase alone had no effect, suggesting that there is considerable redundancy. 